\title{
Sistema de Información para Detección de Crecientes Súbitas en la Cuenca del Río Manzanares en Santa Marta, Colombia
}

\author{
Wilmer Quintero(1), Carlos A. Robles ${ }^{(2,1)^{*}}$ y Adolfo M. Viloria ${ }^{(1)}$ \\ (1) Facultad de Ingeniería, Universidad Cooperativa de Colombia. Troncal del Caribe, Sector Mamatoco, \\ Santa Marta-Colombia (e-mail: wilmer.quintero@outlook.com; viloria.adolfo@gmail.com) \\ (2) Facultad de Ingeniería, Universidad del Magdalena. Carrera 32 No. 22 - 08, Santa Marta-Colombia \\ (e-mail: carlosarturo.ing@gmail.com)
}

${ }^{*}$ Autor a quien debe ser enviada la correspondencia

Recibido May. 29, 2017; Aceptado Jul. 26, 2017; Versión final Ago. 15, 2017, Publicado Dic. 2017

\begin{abstract}
Resumen
En este artículo se presenta un sistema de información que procesa los datos de equipos instalados para monitorear la humedad del suelo, precipitación y nivel de agua del río Manzanares como parte de un programa de alerta temprana de creciente súbita en la Cuenca del Río Manzanares en Santa Marta, Colombia. El sistema detecta crecientes súbitas y emite tres tipos de alerta, amarilla, naranja y roja, dependiendo del nivel del río. Para el desarrollo tecnológico se utilizó Java Enterprise Edition en conjunto con interfaces de programación como Google Maps, Google Cloud Messaging, Google Play Services y una base de datos relacional para almacenar la información. Los resultados demuestran la importancia de los sistemas de información como herramienta para alertar a las comunidades en riesgo de inundación.
\end{abstract}

Palabras clave: sistema de información; interfaz de programación; sistema de alerta temprana; riesgo de inundaciones; plataforma Java

\section{Information System for Flash Flood Detection in the Manzanares River Basin in Santa Marta, Colombia}

\begin{abstract}
This paper presents an information system that processes the installed equipment data to monitor soil moisture, precipitation and water level of the Manzanares River, as part of a program for the early warning of flash flood in the Manzanares River Basin in Santa Marta, Colombia. The system detects flash floods and emits three types of alert, yellow, orange and red, depending on the level of the river. For technological development, Java Enterprise Edition was used, in conjunction with programming interfaces such as Google Maps, Google Cloud Messaging, Google Play Services and a relational database to store information. The results demonstrate the importance of information systems as a tool to alert communities at risk of flooding.
\end{abstract}

Keywords: information system; programming interface; early warning system; flood risk; Java platform enterprise edition 


\section{INTRODUCCIÓN}

Los desastres naturales como las inundaciones son fenómenos mundiales que ocurren cada vez con más frecuencia y producen pérdidas de gran magnitud, especialmente en las comunidades vulnerables donde los impactos que se generan sobre la población y sus bienes son cada vez más importantes y perjudiciales (Sedano et al., 2013; Ávila et al., 2016). En el contexto regional se puede observar que el río Manzanares en Santa Marta Colombia, a través de su recorrido representa una gran amenaza puesto que en temporadas de fuertes lluvias se presentan crecientes súbitas que afectan a las comunidades aledañas al río, así como avenidas, cementerios, entre otras edificaciones (Caracol, 2016).

Las crecientes súbitas del río ocasionan inundaciones a lo largo de la parte baja del cauce, afectando aproximadamente a 300 familias de barrios como Salamanca, 8 de febrero, Pamplonita, Villa del Río, Villa U, María Eugenia, La Esmeralda, Las Malvinas, 20 de enero y un sector de Villa del Carmen (El Tiempo, 2010). Estas inundaciones ocasionan daños en los mobiliarios y electrodomésticos de los habitantes, los cuales en muchas ocasiones no tienen tiempo para reaccionar ante las crecientes del río y no logran aplicar las medidas correctivas para poner a salvo sus pertenencias. De igual forma sucede con los organismos de atención que no alcanzan a actuar oportunamente ante este tipo de eventos. En este contexto, toman relevancia los sistemas de alerta temprana (SAT) como un mecanismo efectivo para dar aviso a las comunidades y organismos de atención con suficiente antelación a la ocurrencia de un evento de inundación. Con este tipo de sistemas se puede alertar a la población de un posible peligro inminente, y también se les brinda una herramienta tecnológica a los organismos de atención para el proceso de gestión del riesgo (Melgarejo y Lakes, 2014; Fakhruddin et al., 2015).

Uno de los principales componentes de los sistemas de alerta temprana lo constituyen los sistemas de información, los cuales deben cumplir con las siguientes características: funcionamiento continuo, soportar momentos de estrés sin pérdida del rendimiento y realizar el proceso de almacenamiento y estudio de datos en el menor tiempo posible (Cools et al., 2016). Estos requerimientos han llevado a que los sistemas de alerta temprana se actualicen e incluyan el procesamiento de datos en servidores dedicados, servicios de la nube y plataformas móviles (Hou et al., 2013). A nivel mundial se han realizado desarrollos orientados a solucionar problemáticas asociadas a las inundaciones, entre las que se destacan: (Mure-Ravaud et al., 2016) realizaron una herramienta web para la ciudad de Dijon Francia cuya función es la predicción de inundaciones, (Kulkarni et al., 2014) desarrollaron un sistema de información para la simulación de inundaciones en ciudades costeras utilizando un modelo de flujo en tierra y (Jagtman, 2010) utilizó la inclusión de los dispositivos móviles para advertir al público de una situación de amenaza.

Con el avance de la tecnología la cantidad de variables físicas ambientales que se pueden recolectar es cada vez mayor, por lo cual se requiere de sistemas con la capacidad de almacenar y consultar grandes volúmenes de información. En el caso colombiano, para cumplir con dicho requerimiento se han implementado centros de procesamiento de datos que cuentan con infraestructura física y equipos informáticos de última tecnología, entre las que se destacan el sistema de gestión de la información ambiental del Instituto de Hidrología, Meteorología y Estudios Ambientales (IDEAM) y el sistema de alerta temprana SIATA de la ciudad de Medellín (Domínguez y Lozano, 2014). Estos sistemas poseen en común que integran un conjunto de actores, políticas, procesos y tecnologías para gestionar información sobre el uso, aprovechamiento y sostenibilidad del ambiente.

Teniendo en cuenta lo anterior, el objetivo de este trabajo consiste en desarrollar un sistema de información que se encarga de recibir los datos provenientes de tres tipos de sensores instalados en la cuenca del río Manzanares para monitorear la humedad del suelo, precipitación y nivel de agua (Guillot et al., 2017); con un periodo de muestreo horario, pero completamente parametrizable. El sistema genera un informe con tres tipos de alertas: amarilla, naranja y roja. El informe puede ser enviado a los entes de gestión del riesgo y ser consultado desde un navegador web o dispositivo móvil con Android. En ese orden de ideas, a continuación, se presenta la metodología utilizada en el trabajo. Se detalla en el patrón de desarrollo utilizado, la plataforma de desarrollo basada en Java, esquema general, esquema de servicio y diagramas de flujos de los principales procesos implementados.

\section{METODOLOGÍA}

En el presente trabajo se utilizó un tipo de investigación aplicada en virtud de que se presenta una solución tecnológica a un problema concreto relacionado con las inundaciones debido al desbordamiento del río Manzanares. Además, teniendo en cuenta que la técnica es un proceso indispensable en la investigación, se utilizaron técnicas de investigaciones documentales y de campo con el fin de dimensionar el problema y desarrollar un sistema de información acorde a la problemática en estudio. En ese orden de ideas, en adelante se presenta la metodología llevada a cabo para el desarrollo del sistema de información. 


\section{Localización del Área de Estudio}

Santa Marta es la capital del departamento del Magdalena con una población de 454,860 habitantes, está ubicada en las estribaciones de la sierra nevada de Santa Marta. Limita al norte y al oeste con el Mar Caribe, al sur con Ciénaga y Aracataca y al oriente con los Departamentos de la Guajira y Cesar. Sus coordenadas geográficas son: $11^{\circ} 14^{\prime} 50^{\prime \prime}$ de latitud norte y $74^{\circ} 12^{\prime} 06^{\prime \prime}$ de latitud oeste. El río manzanares atraviesa toda la ciudad, nace en la vertiente noreste de la Sierra Nevada en la cuchilla de San Lorenzo a $2300 \mathrm{msnm}$ y desemboca en el Mar Caribe en la playa de los cocos de la bahía de Santa Marta. Cuenta con $174.54 \mathrm{~km}^{2}$ de superficie, $70.34 \mathrm{~km}$ de perímetro y $33.50 \mathrm{~km}$ de largo en su cauce principal (Figura 1)

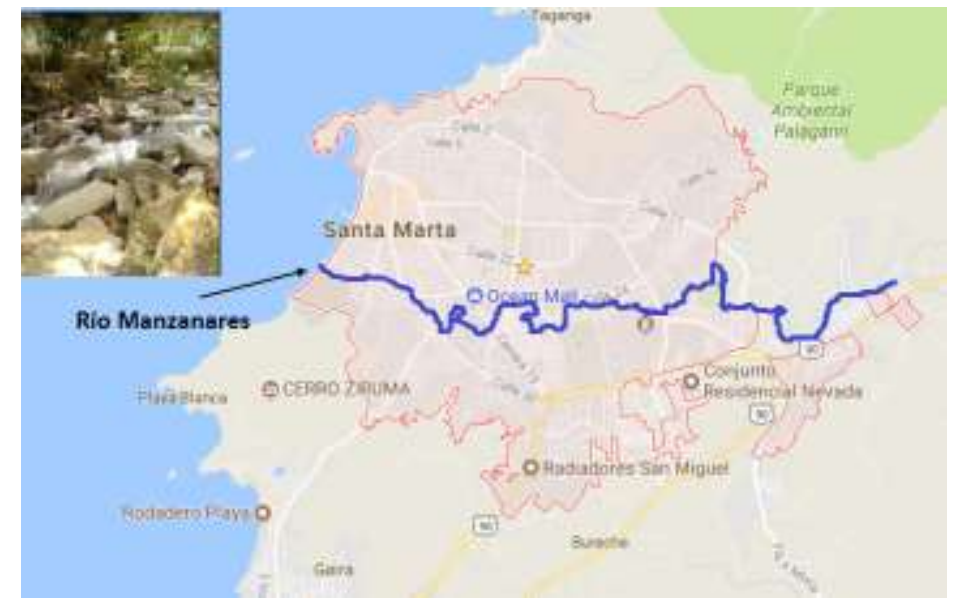

Fig.1: Ubicación del río Manzanares.

\section{Metodología Scrum}

Para el desarrollo del proyecto se utilizó la metodología de desarrollo ágil Scrum, la cual permite adoptar una estrategia de desarrollo iterativa e incremental en la que se realizan segmentación de tareas, asignación de roles y seguimiento diario de los avances. Durante cada iteración se realiza una entrega parcial del software con el fin de ajustar las funcionalidades desarrolladas y agregar nuevas prestaciones (Gómez et al., 2016; Nidagundi y Novickis, 2017). En la figura 2 se muestra el esquema general de esta metodología de desarrollo, en la cual se inicia con la definición de un listado de productos o requisitos y se retroalimenta a través de la prospectiva que se realiza al final de cada iteración.

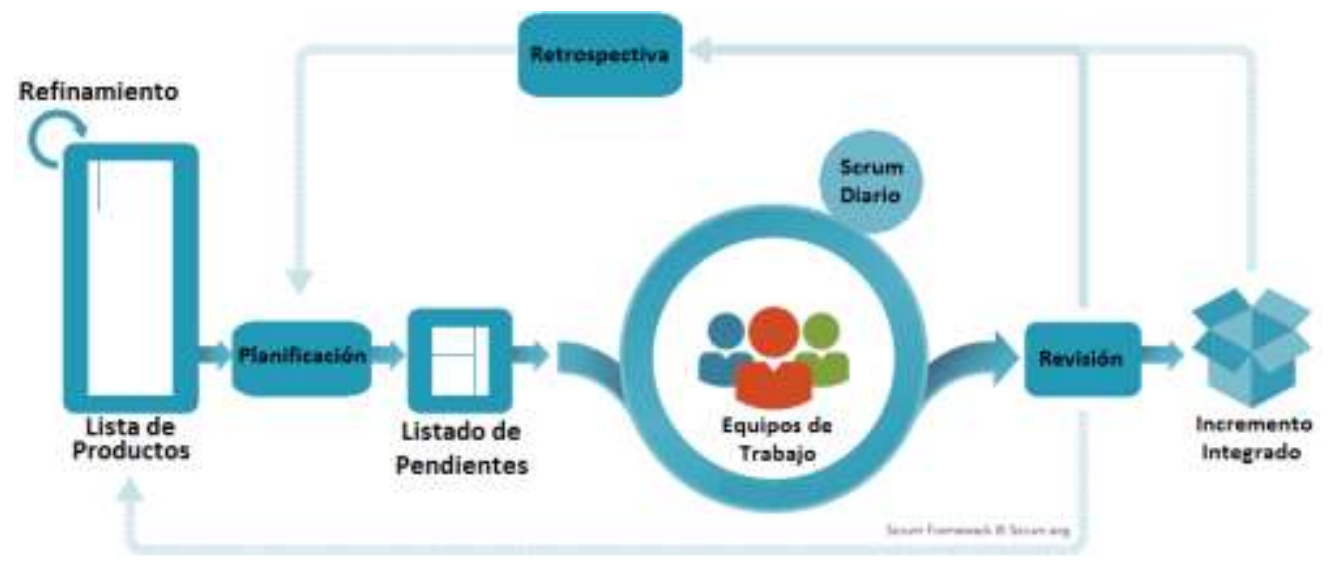

Fig.2: Estructura de trabajo SCRUM. (Adaptada de Scrum, 2017)

\section{Arquitectura}

Se utilizó el modelo vista controlador (MVC) dado que los requerimientos crean un escenario favorable para esta arquitectura donde los datos deben ser recibidos, almacenados y estudiados en un solo lugar, en este caso un servidor centralizado. La característica principal de MVC es la separación de los datos y la lógica del negocio de la interfaz del usuario, con lo cual se logra una comunicación agradable con el software. La lógica del negocio se refiere todo el procesamiento de la información y captura de los datos de las distintas capas del software (Alarcón et al., 2016). El modelo se encarga de persistir los datos, del controlador donde se aplica la lógica de negocio y de la presentación que es la capa que se le muestra al cliente. 


\section{Plataforma de Desarrollo}

La plataforma de desarrollo utilizada fue Java Enterprise Edition, la cual se integra al modelo MVC sin causar ningún tipo de conflicto. Esta plataforma incorpora un sin número de capas distribuidas y un conjunto de características únicas como lo son los Enterprise JavaBeans, Servlets y la posibilidad de utilizar diferentes interfaces de programación (API) como servicios web java para XML (JAX-WS), conectividad a base de datos Java (JDBC), invocación remota de métodos (RMI) y distintos tipos de API para la web como lo son JavaServer Faces (JSF) o JavaServer Pages (JSP) (Figura 3)

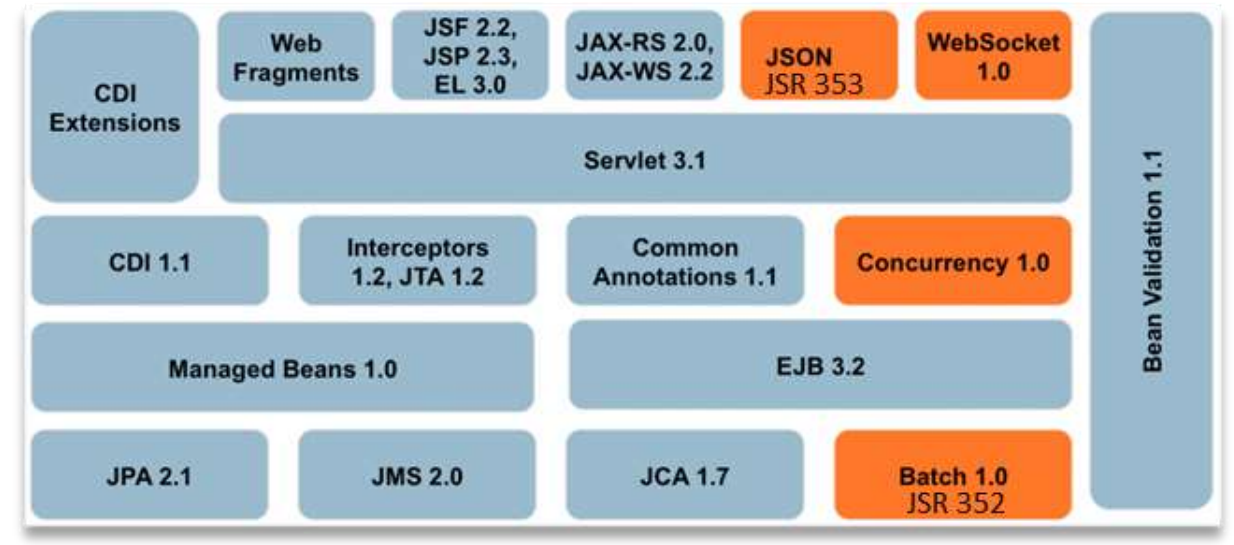

Fig. 3: Plataforma de desarrollo Java EE. (Adaptada de Java, 2017)

Se desarrolló una aplicación móvil en el sistema operativo Android que permite enviar las alertas a las entidades encargadas del riesgo. En la figura 4 se representa la forma en la que interactúan los diferentes componentes del sistema de información. El web service se encarga de recibir la información, Java Beans contiene la inteligencia del negocio y la persistencia se encarga del almacenamiento de los datos.

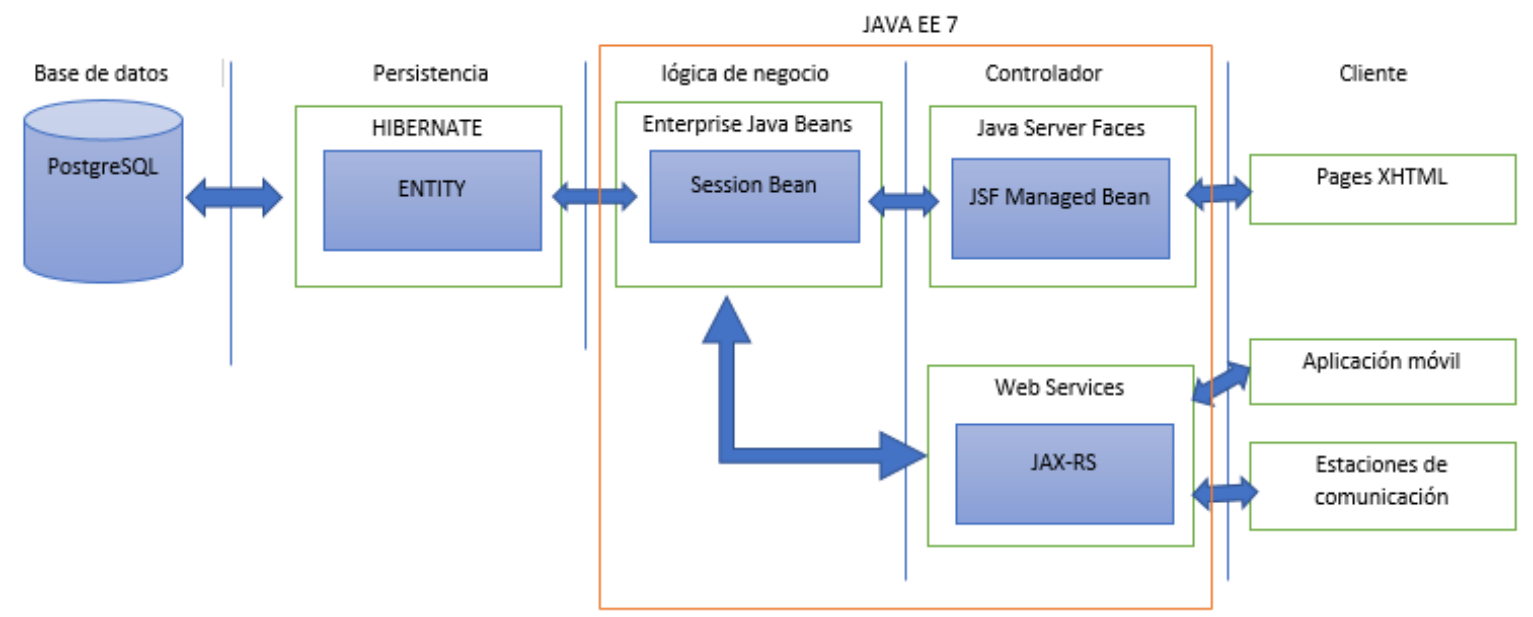

Fig. 4: Esquema general del sistema construido

Dentro de las principales funciones del sistema desarrollado se pueden enumerar las siguientes: 1) recepción de datos desde las estaciones de trasmisión; 2) generación de un reporte que es enviado vía correo electrónico a las personas encargadas; 3) notificaciones inmediatas al aplicativo móvil; y 4) envío de alertas de nuevos incidentes a las entidades encargadas de la gestión del riesgo a través de la aplicación móvil "Está Sucediendo". Todos estos procesos están encaminados a la reducción del tiempo desde el momento que el evento empieza a suceder hasta que las entidades se enteran de lo que sucede. En la figura 5.a se describe el proceso de adquisición de datos desde la estación de trasmisión. En la figura 5.b se muestra el proceso general para enviar un reporte.

Para la realizar la comunicación entre la estación de trasmisión y el servidor se utilizó la red GSM o 3G dependiendo de la disponibilidad del proveedor del servicio. Para la trasmisión de datos se utilizó un web services de tipo rest, el cual recibe información en texto plano a través de una URL: http://181.143.200.174:8080/sipnat/webresources/Sensor/"id"/"variable"/"fecha". Dependiendo del resultado de la petición, el servidor responderá con el digito 2 en el caso que exista un resultado positivo, mientras que responderá con el 1 en caso que se genere un error con la recepción de datos. 

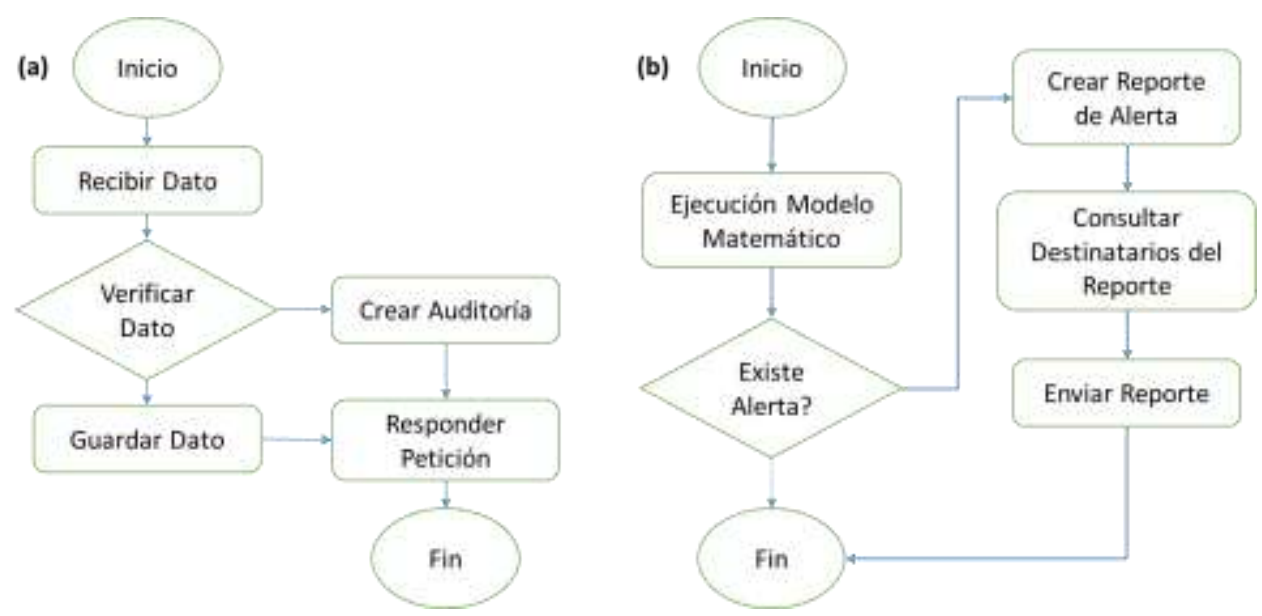

Fig. 5: (a) Recepción de información. (b) Reporte de alerta

Adicionalmente, se desarrolló una aplicación móvil SIPNAT creada para recibir alertas y conocer los datos que se encuentran en la base de datos del servidor. Se utilizó la misma arquitectura de web services rest; pero utilizando el método POST para el envío de información. Además, se trasmite un componente de formato de texto ligero (JSON) que fue creado para la optimización y el intercambio de información legible, el cual es fácil de encriptar y desencriptar. Para emitir los mensajes de alerta se utilizaron un conjunto de servicios y API's como Google Cloud Messaging (GCM) recientemente actualizada a Firebase Cloud Messaging (FCM). Esta interfaz de programación permite enviar de forma gratuita un archivo GSON con peso máximo de 4KB de manera instantánea a cualquier dispositivo Android y/o IOS (Schwarz et al., 2013). La ventaja de utilizar este tipo de interfaz radica en que no se requiere que la aplicación esté en funcionamiento en el dispositivo para que reciba la información (Figura 6).

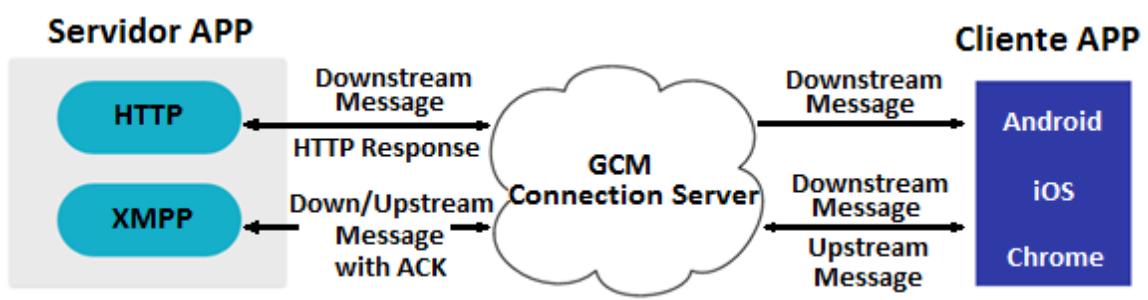

Fig. 6: Esquema básico de servicio GCM. (Adaptada de Google, 2016)

La comunicación de la alerta se realiza a través del aplicativo Android y a través de un correo electrónico utilizando la API JavaMail. El correo es enviado a las entidades encargadas de la gestión de riesgo del distrito de Santa Marta en el instante que el sistema detecta un cambio de nivel en el río. Utilizando la librería de código abierto JasperReports se crea un archivo pdf con la información generada por el sistema, con el fin de adjuntarlo al correo enviado a los entes de gestión del riesgo de la ciudad. Finalmente, para la visualización de mapas tanto en el aplicativo web como en el aplicativo móvil se utilizó Google Maps.

\section{Metodología para evaluar el aplicativo}

Con el fin de evaluar la funcionalidad del aplicativo, se realizaron pruebas con los datos provenientes de los sensores de nivel del agua. Se trasmitieron 60 datos desde el sensor con valores entre $0 \mathrm{~cm}$ y $400 \mathrm{~cm}$. Un valor obtenido entre $0 \mathrm{~cm}$ y $200 \mathrm{~cm}$ corresponde a un estado normal, entre $200 \mathrm{~cm}$ y $300 \mathrm{~cm}$ se genera un cambio de estado a alerta amarilla, entre $300 \mathrm{~cm}$ y $400 \mathrm{~cm}$ se emite una alerta naranja y para valores mayores que $400 \mathrm{~cm}$ se tiene alerta roja. Estos niveles están bajo estudio y se ajustarán de acuerdo a los resultados finales que se obtengan con el estudio topográfico e hidráulico del caudal del río Manzanares.

\section{RESULTADOS Y DISCUSIÓN}

Se desarrolló un sistema completamente escalable que permite la inclusión de nuevas cuencas independientemente de su localización geográfica. Se pueden crear diferentes proyectos de medición de parámetros ambientales dado que la plataforma permite la integración de equipos de medios de terceros. De igual forma, es posible parametrizar aspectos como la ubicación de los sensores y la unidad de medida como se observa en la figura 7. En la sección Tipo de Sensor se pueden crear los sensores al marcar la casilla correspondiente. Una vez se marca un tipo de sensor, aparecerán los sensores creados en el recuadro Sensor ya existente. La ubicación de los sensores se puede ver en el mapa utilizando el icono para visualizar. En el 
recuadro Sensor, se puede editar de manera exacta la ubicación de un nuevo sensor y hacer una breve descripción. En los Datos del proyecto se ingresa el nombre y descripción del proyecto. Finalmente, en el recuadro de Sensores asignados aparecerán los sensores asignados al proyecto que se encuentra en proceso de creación.

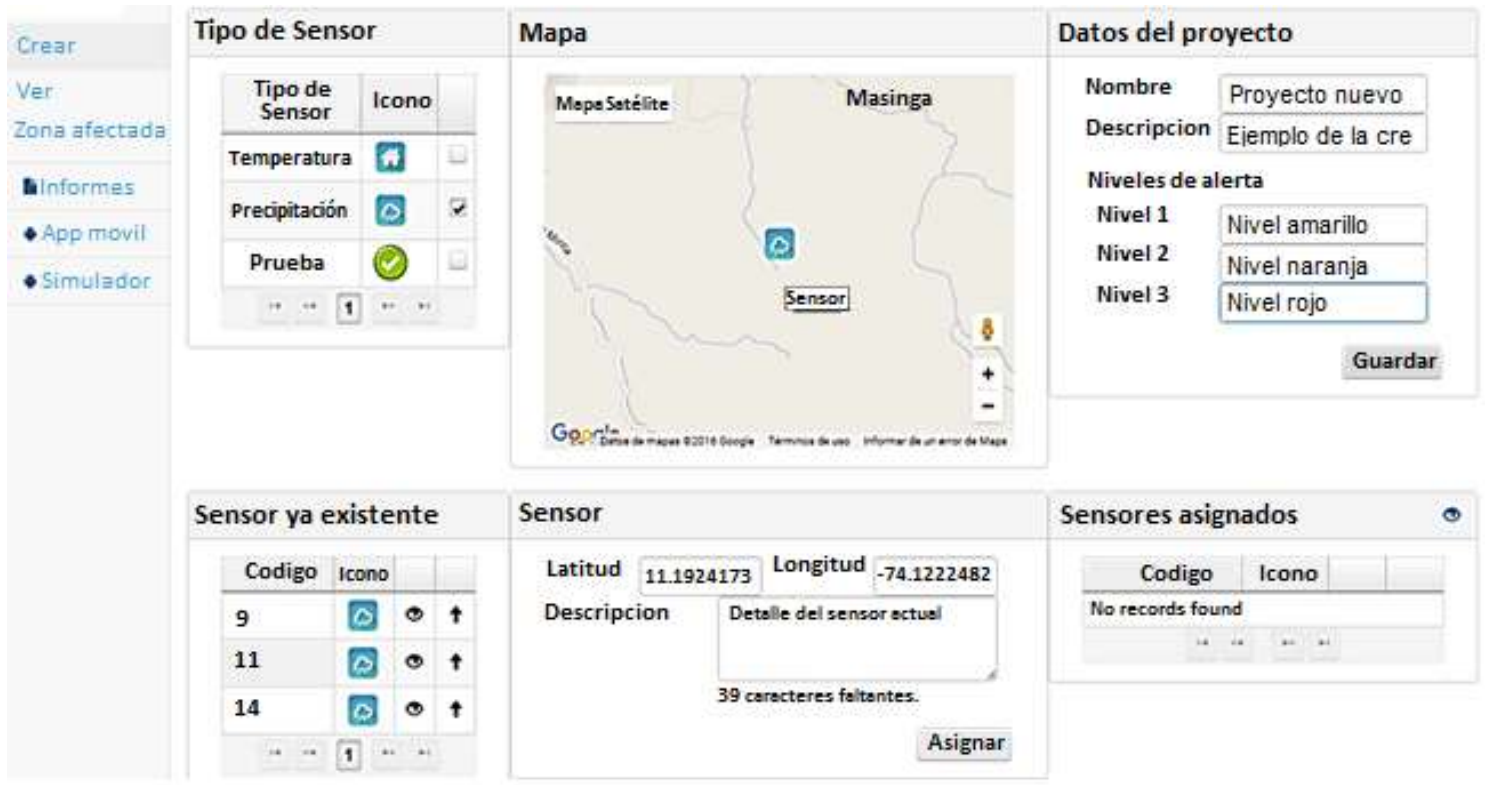

Fig. 7: Esquema general para la creación de un proyecto

En el aplicativo móvil, el cual es una extensión del sitio web, se permite la visualización de los proyectos de medición y los sensores en el mapa (ver figura 8.a). Cuando se selecciona alguno de los sensores se muestran los datos de las últimas 24 horas como se puede ver en la figura 8.b. La gran utilidad del aplicativo es que además de poder observar la información que el servidor recibe de los sensores, puede enviar notificaciones de alerta en tiempo real a dispositivos móviles. Cuando existen variaciones en los datos se genera una notificación al aplicativo móvil informando del cambio de nivel de alerta. Además, se realiza el envío de una circular a las entidades encargadas de la gestión y manejo del riesgo en el distrito de Santa Marta para que se tomen las medidas pertinentes y se cuente con el tiempo necesario para aplicar los protocolos indicados. En la figura 8.c se puede ver el resultado obtenido cuando se emite una alerta roja.
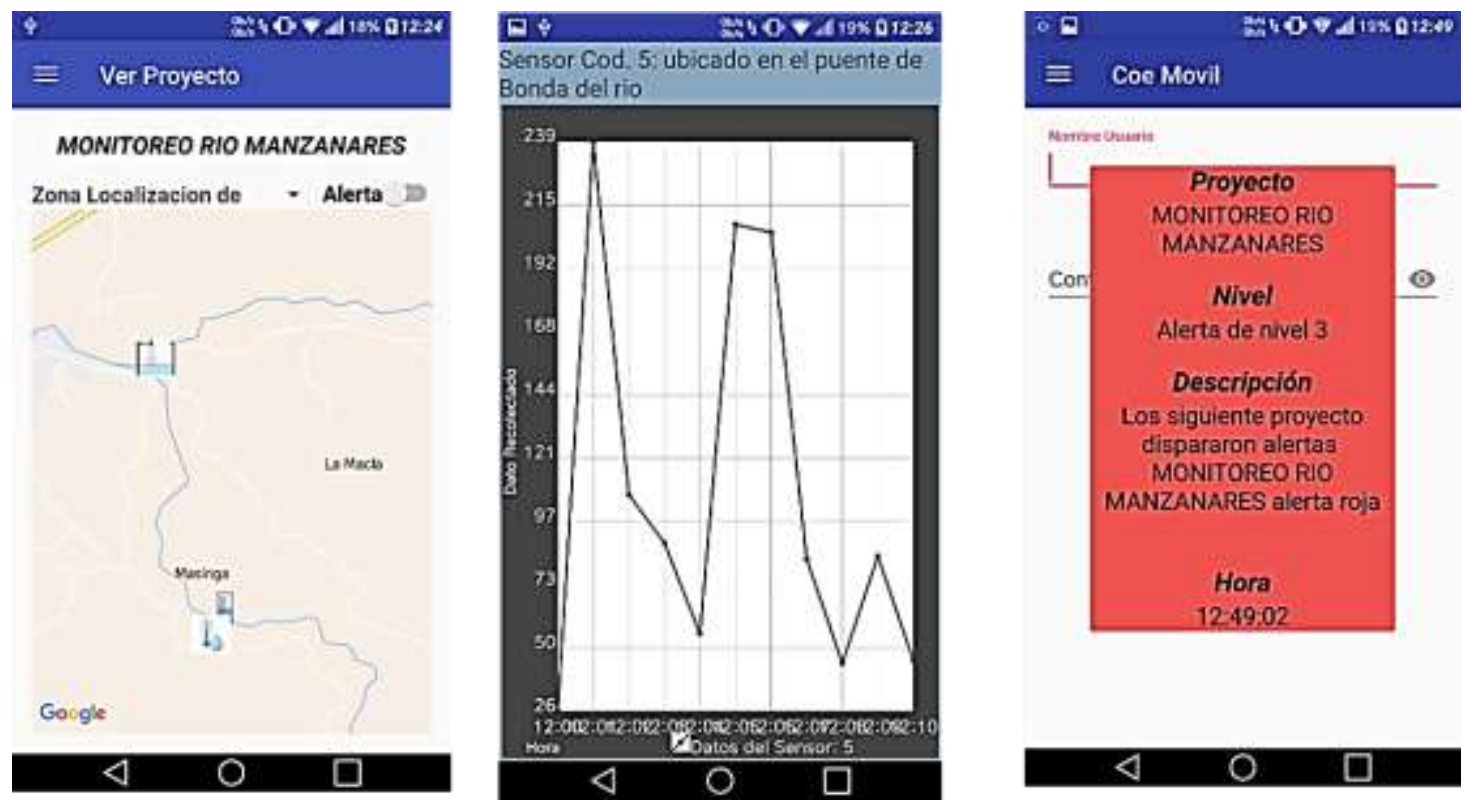

Fig. 8: (a) Sensores en la aplicación móvil. (b) Datos de un sensor. (c) Modelo para alerta roja

En este punto es importante mencionar que el sistema desarrollado tomó como referencia el proyecto implementado por la corporación autónoma regional de la Guajira en la sierra nevada de Santa Marta Colombia, el cual cuenta con un sistema de información para el monitoreo de variables ambientales proporcionadas por las estaciones de medición (Corpoguajira, 2014). En el mismo sentido, esta investigación guarda relación con lo presentado por (Carrillo et al., 2016) en cuanto a la estructura de desarrollo utilizada. 
Adicionalmente, en esta investigación se utilizó el enfoque basado en sistemas del SIATA, es decir, que el sistema general se dividió en varios subsistemas con una posterior integración de todos los procesos (Domínguez y Lozano, 2014).

Por otra parte, es de mencionar el trabajo realizado por (Gómez et al., 2016), en el cual desarrollaron un sistema de información con aplicación en el sector agrícola haciendo uso de la metodología Scrum, pero utilizando una plataforma de desarrollo diferente a la trabajada en el presente trabajo. Lo anterior deja en evidencia la importancia de este tipo de metodologías que pueden ser aplicadas a cualquier tipo de proyecto de software obteniendo resultados óptimos. También es de resaltar los resultados de la investigación de (Wang et al., 2015) quienes realizaron una aplicación móvil para el monitoreo de cuencas en pequeños ríos; la cual a diferencia del presente trabajo genera reportes de incidentes diferentes a las inundaciones.

Teniendo en cuenta lo anterior, es de resaltar que el sistema desarrollado en esta investigación tiene como características novedosas que abordó el tema de multi-tenencia haciendo principal énfasis en la robustez y utilidades que se logran al utilizar una capa de presentación basada en Java server pages. Adicionalmente, se utilizó la mensajería push para alertar en tiempo real a las entidades responsables de la gestión del riesgo sin la necesidad de que este tipo de organismos estén presentes en un centro de monitoreo.

\section{CONCLUSIONES}

A partir de los resultados obtenidos con el sistema de información, se derivan las siguientes conclusiones:

1.- Se desarrolló un sistema de información haciendo uso de nuevas tecnologías y una arquitectura de trabajo robusta basada en el modelo vista controlador, con el cual se pueden realizar tres tipos de alerta: amarilla, naranja y roja; para beneficiar a una población de aproximadamente 300 familias en riesgo de inundación debido a desbordamientos en la cuenca del río Manzanares. Además, el sistema es completamente parametrizable y se puede extender a otras cuencas que no cuentan con un sistema de alerta temprana.

2.- La elección de Java Enterprise Edition fue acertada puesto que permitió una integración con las diferentes interfaces complementarias necesarias para el desarrollo del proyecto como lo son Google Maps, Jasper Report y Java mail. Además, con la utilización de las diferentes tecnologías de código abierto se desarrolló un proyecto robusto basado en herramientas que no incrementan su costo de implementación directo.

3.- Se logró desarrollar una aplicación móvil que permite generar reportes de los parámetros ambientales y niveles de alertas obtenidos. De igual forma, la utilización de la arquitectura de web services rest permite la generación de reportes relacionados con otro tipo de riesgos para futuros proyectos.

4.- Dentro de las pruebas de conectividad y de funcionalidades del sistema, se consiguieron los resultados esperados. El servidor no superó el $20 \%$ de su capacidad, mientras que la velocidad de la conexión a internet proporcionó un ambiente de funcionamiento ideal para el proyecto.

5.- El código del proyecto se manejó a través de un controlador de versiones tipo GIT, el cual facilitó de gran manera el trabajo en equipo. Además, se tiene la posibilidad de crear nuevos brazos para la implementación de nuevas funcionalidades y/o actualizaciones diferentes a las que se encuentran desarrolladas.

\section{AGRADECIMIENTOS}

Los autores agradecen al Departamento Administrativo de Ciencia, Tecnología e Innovación de Colombia (Colciencias) por la financiación del proyecto Sistema de Alerta Temprana para Gestionar el Riesgo por Inundación en la Cuenca del Río Manzanares, con recursos del patrimonio autónomo fondo nacional de financiamiento para la ciencia, la tecnología y la innovación, Francisco José de Caldas.

\section{REFERENCIAS}

Alarcón, A., Urrutia, J. y Callejas, M., Aplicación Móvil para la Administración de Variables Físicas en Ciclismo al Aire Libre, doi: 10.4067/S0718-07642016000400019, Inf. Tecnol. (en línea), 27(4), 175-182 (2016)

Ávila, A., Justino, F., Aaron, W., Bromwich, D. y Amorim, M., Recent precipitation trends, flash floods and landslides in southern Brazil, doi: 10.1088/1748-9326/11/11/114029, Environ. Res. Lett., (en línea), 11, 1-13 (2016)

Caracol, Fuerte lluvia causa estragos en Santa Marta. https://goo.gl/4xheb2. Acceso: 04 de agosto 2017. Caracol Radio (2016) 
Carrillo, M., Franky, C., Páez, P. y Andrés F., SCLOUDPY: Sistema Informático Web de Multi-Tenencia para el procesamiento en la Nube de Pedidos de PYMES, doi: 10.4067/S0718-07642016000100019, Inf. Tecnol. (en línea), 27(1), 181-194 (2016)

Cools, J., Innocenti, D. y O'Brien, S., Lessons from flood early warning systems, doi: 10.1016/j.envsci.2016.01.006, Environ Sci \& Policy, (en línea), 58, 117-122 (2016)

Corpoguajira, Sistema de alerta temprana (SAT). https://goo.gl/FRgq6K. Acceso: 11 de agosto 2017. Corpoguajira (2014)

Domínguez, E. y Lozano, S., Estado del arte de los sistemas de alerta temprana en Colombia, Rev. Acad. Colomb. Cienc, ISSN: $0370-3908$ (en línea), 38(148), 321-332, 2014. https://goo.gl/9Fgnnd. Acceso: 11 de Agosto (2017)

El Tiempo, 300 familias afectadas por inundaciones en Santa Marta (Magdalena). https://goo.gl/p7KMNc. Acceso: 04 de agosto 2017), El Tiempo (2010)

Fakhruddin, S.H., Kawasaki, A. y Babel, M.S., Community responses to flood early warning system: Case study in Kaijuri Union, Bangladesh, doi: 10.1016/j.ijdrr.2015.08.004, Int. J. Disaster Risk Reduction, (en línea), 14(4), 323-331 (2015)

Gómez, U., Pérez, J. y Ramírez, J., Sistema de Información Agrícola para la disminución de Brechas entre Oferta y Demanda - AGROCRAFT, doi: 10.4067/S0718-07642016000300020, Inf. Tecnol. (en línea), 27(3), 215-220 (2016)

Google, Google developers (en línea), https://goo.gl/vfk4db. Acceso: 11 de Abril (2017), Google Cloud Messaging, USA (2016)

Guillot, J., Robles, C. y Callejas, J., Adquisición de Señales Ambientales para un Sistema de Alerta Temprana, Inf. Tecnol. 28(5), en prensa (2017)

Hou, S., Li, A., Han, B. y Zhou, P., An Early Warning System for Regional Rain-Induced Landslide Hazard, doi: 10.4236/ijg.2013.43053, Int. J. Geosci, (en línea), 4, 584-587 (2013)

Jagtman, H., Cell broadcast trials in The Netherlands: Using mobile phone technology for citizens' alarming, doi: 10.1016/j.ress.2009.07.005, Reliab Eng Syst Safe, (en línea), 95(1), 18-28 (2010)

Java, Java Platform (en línea), https://goo.gl/cqCLBN. Acceso: 7 de Abril (2017), Oracle, USA (2017)

Kulkarni, A., Mohanty, J., Eldho, T., Rao, E. y Mohan, B., A web GIS based integrated flood assessment modeling tool for coastal urban watersheds, doi: 10.1016/j.cageo.2013.11.002, Comput Geosci, (en línea), 64, 7-14 (2014)

Melgarejo, L. y Lakes, T., Urban adaptation planning and climate-related disasters: An integrated assessment of public infrastructure serving as temporary shelter during river floods in Colombia, doi: 10.1016/j.ijdrr.2014.05.002, Int. J. Disast Risk Re, (en línea), 9, 147-158 (2014)

Mure-Ravaud, M., Binet, G., Bracq, M., Perarnaud, J. y Fradin, A., A web based tool for operational real-time flood forecasting using data assimilation to update hydraulic states, doi: 10.1016/j.envsoft.2016.06.002, Environ Modell Softw, (en línea), 84, 35-49 (2016)

Nidagundi, P. y Novickis, L., Introducing Lean Canvas Model Adaptation in the Scrum Software Testing, doi: 10.1016/j.procs.2017.01.078, Procedia Comput Sci, (en línea), 104, 97-103 (2017)

Schwarz, R., Dutson, P., Steele, J. y To, N., The Android Developer's Cookbook: Building Applications with the Android SDK, 2 ${ }^{\mathrm{a}}$ Ed., 349-359. Addison-Wesley, Boston, USA (2013)

Scrum, Scrum (en línea), https://goo.gl/yXGT3x. Acceso: 14 de Abril (2017), Scrum.org, USA (2017)

Sedano, K., Carvajal, Y. y Ávila, A., Análisis de aspectos que incrementan el riesgo de inundaciones en Colombia, Rev. Luna Azul, ISSN: 1909-2474 (en línea), 37, 219-238, 2013. https://goo.gl/wYwmU2. Acceso: 3 de Agosto (2017)

Wang, Y., Zhang, W., Engel, B., Peng, H., Theller, L., Shi, Y. y Shan Hu., A fast mobile early warning system for water quality emergency risk in ungauged river basins, doi: 10.1016/j.envsoft.2015.08.003, Environ Modell Softw, (en línea), 73, 76-89 (2015) 